Gut and Liver, Vol. 11, No. 3, May 2017, pp. 319-320

\title{
Disability Index as a New Outcome Measure in Patients with Inflammatory Bowel Disease
}

\author{
Hyun $\mathrm{Ho} \mathrm{Choi}^{1}$ and Young-Seok $\mathrm{Cho}^{2}$
}

${ }^{1}$ Department of Internal Medicine, Uijeongbu St. Mary's Hospital, College of Medicine, The Catholic University of Korea, Uijeongbu, and ${ }^{2}$ Department of Internal Medicine, Seoul St. Mary's Hospital, College of Medicine, The Catholic University of Korea, Seoul, Korea

See "Disability due to Inflammatory Bowel Disease Is Correlated with Drug Compliance, Disease Activity, and Quality of Life" by Jin Young Yoon, et al. on page 370, Vol. 11. No. 3, 2017

Inflammatory bowel diseases (IBDs), including Crohn's disease (CD) and ulcerative colitis (UC), are idiopathic inflammatory disorders of the gastrointestinal tract. IBDs are chronic and disabling conditions that negatively affect the physical, psychological, familial and social dimensions of life. ${ }^{1}$ The clinical diagnosis and assessment of IBD are based on clinical symptoms and signs as well as laboratory, endoscopic, and histologic findings. ${ }^{2}$ These parameters are used to evaluate the activity of the disease at a given time. However, the assessment of long-term disease severity is important for the guidance of decision making, because the natural history of IBD is characterized by periods of relapse and remission, potentially leading to long-term complications requiring repeated hospitalizations and interventions. ${ }^{3}$ Over the past few decades, health-related quality of life (HRQoL) measurements have been used to determine the range of functional limitations in patients with IBD. ${ }^{4}$ However, HRQoL is a subjective assessment of patients' feelings on their limitations and restrictions. Specific tools, such as the Inflammatory Bowel Disease Questionnaire (IBDQ), have not been approved as valid by the U.S. Food and Drug Administration (FDA). ${ }^{5}$

A disability (or function decrement) is the result of an interaction between an underlying disease or health condition and contextual factors, specifically environmental and personal factors. ${ }^{6}$ Disability is an objective concept referring to the problems that a patient may have in different health domains. ${ }^{6}$ Recently, an international group of experts working in collaboration developed the first Inflammatory Bowel Disease Disability Index (IBD-DI) to measure the functional consequences and disease burden of IBD according to categories of the International
Classification of Functioning, Disability and Health, from the World Health Organization. ${ }^{6}$ A prospective validation study in Australia that involved 166 patients with IBD demonstrated that the IBD-DI may be a valid tool for measuring disability, as it showed good correlations with the Crohn's Disease Activity Index (CDAI), partial Mayo score (pMayo), and the IBDQ. In addition, scores on this index were correlated with workforce participation, suggesting its utility for assessment of restrictions on participation and limitations of activity. ${ }^{7}$ In a more recent study, the French version of the IBD-DI (with 14 items whose total score ranges from 0 to 100) was reported to be a useful tool for measuring disability, with high internal consistency, interobserver reliability, and construct validity in a populationbased cohort of French patients with IBD. The risk factors for high IBD-DI were female sex, high clinical disease activity, and short ( $<8$ years) or long ( $\geq 17$ years) disease duration. ${ }^{1}$ These results suggest that IBD-DI can be used as an outcome measure in clinical trials and prospective epidemiological studies. ${ }^{1,7}$

In this issue of Gut and Liver, the article "Disability due to inflammatory bowel disease is correlated with drug compliance, disease activity, and quality of life" by Yoon et al. ${ }^{8}$ used the Korean version of the IBD-DI, which was developed using forward/ backward translation, and evaluated its correlation with other parameters, including clinical disease activity indices (CDAI and pMayo) and the Crohn's and Ulcerative Colitis Questionnaire-8 for disease-specific QoL. The results showed that the IBD-DI was strongly correlated with the disease activity index and HRQoL of Korean patients with IBD. In addition, better drug compliance was associated with lower disability and higher QoL, emphasiz-

Correspondence to: Young-Seok Cho

Department of Internal Medicine, Seoul St. Mary’s Hospital, College of Medicine, The Catholic University of Korea, 222 Banpo-daero, Seocho-gu, Seoul 06591, Korea

Tel: +82-2-2258-6021, Fax: +82-2-2258-2038, E-mail: yscho@catholic.ac.kr pISSN 1976-2283 eISSN 2005-1212 https://doi.org/10.5009/gnl17103

@ This is an Open Access article distributed under the terms of the Creative Commons Attribution Non-Commercial License (http://creativecommons.org/licenses/by-nc/4.0) which permits unrestricted non-commercial use, distribution, and reproduction in any medium, provided the original work is properly cited. 
ing the importance of medication compliance for IBD patients.

Although the rate of increase in IBD prevalence varies greatly in Asia, several countries in East Asia have experienced a significant increase in IBD incidence in the past two decades. ${ }^{9}$ In South Korea, the incidences of UC and CD are 4.60 and 3.20 per 100,000 persons, respectively. ${ }^{9}$ Although they suffer from the same condition, the phenotypes, behaviors, complications, and even treatment responses in Asian IBD patients can be different from those of their Western counterparts. Nevertheless, the lives and social activities of IBD patients in Asia are similar to those of Western sufferers, and they are affected by the deterioration of their QoL due to IBD and its symptoms. ${ }^{10}$

Three main domains are used to assess the severity of IBD: the impact of the disease on the patient (clinical symptoms, patient-reported outcomes [PROs], HRQoL, and disability), disease burden (extent, location, and severity of bowel involvement at a given point in time), and disease severity. ${ }^{3}$ Among these, a PRO is defined as a direct report from a patient on his or her health condition or treatment without interpretation by a clinician or anyone else. ${ }^{2}$ The level of interest in PROs in IBD has increased recently. PROs may become required end points for the approval of medical products, which would enhance their safety and effectiveness. ${ }^{2,3,5}$ In addition, PROs could be used to evaluate health services or economic burdens or to assist decision making in routine clinical practice. ${ }^{2}$

The U.S. FDA is moving from composite indices for IBD (e.g., CDAI) to PROs and objective measures of IBD inflammation, such as endoscopy findings. ${ }^{2,5}$ A number of IBD-specific PRO tools-including the IBDQ, the Manitoba IBD Index, the numeric rating scale, the IBD-Control Questionnaire, and the IBDDI-have been developed and validated to assess the impact of disease on outcomes reported by IBD patients. ${ }^{3}$ However, none of these tools meet FDA criteria for development of a valid PRO, because the items were selected from interviews with both patients and healthcare professionals. ${ }^{2,3,5}$

The article by Yoon et al. ${ }^{8}$ is the first report in Korea that IBD-DI can be used as an outcome measure in IBD patients and clinical trials, and it generated results similar to those of Western studies. However, this study did not evaluate changes in the level of disability of Korean patients with IBD over time. In addition, the Korean version of the IBD-DI should be further validated and its reliability evaluated in healthy controls and IBD patients in general gastroenterology community practice. International consensus is required to develop validated PROs according to FDA criteria, and these PROs should be translated into as many languages as possible. ${ }^{5}$ Finally, such PROs could improve the lives of patients with IBD.

\section{CONFLICTS OF INTEREST}

No potential conflict of interest relevant to this article was reported.

\section{REFERENCES}

1. Gower-Rousseau C, Sarter H, Savoye G, et al. Validation of the Inflammatory Bowel Disease Disability Index in a population-based cohort. Gut 2017;66:588-596.

2. Bojic D, Bodger K, Travis S. Patient reported outcome measures (PROMs) in inflammatory bowel disease: new data. J Crohns Colitis 2017;11(Suppl 2):S576-S585.

3. Peyrin-Biroulet L, Panés J, Sandborn WJ, et al. Defining disease severity in inflammatory bowel diseases: current and future directions. Clin Gastroenterol Hepatol 2016;14:348-354.e17.

4. Alrubaiy L, Rikaby I, Dodds P, Hutchings HA, Williams JG. Systematic review of health-related quality of life measures for inflammatory bowel disease. J Crohns Colitis 2015;9:284-292.

5. Williet N, Sandborn WJ, Peyrin-Biroulet L. Patient-reported outcomes as primary end points in clinical trials of inflammatory bowel disease. Clin Gastroenterol Hepatol 2014;12:1246-1256.e6.

6. Peyrin-Biroulet L, Cieza A, Sandborn WJ, et al. Development of the first disability index for inflammatory bowel disease based on the international classification of functioning, disability and health. Gut 2012;61:241-247.

7. Leong RW, Huang T, Ko Y, et al. Prospective validation study of the International Classification of Functioning, Disability and Health score in Crohn's disease and ulcerative colitis. J Crohns Colitis 2014;8:1237-1245.

8. Yoon JY, Shin JE, Park SH, Park DI, Cha JM. Disability due to inflammatory bowel disease is correlated with drug compliance, disease activity, and quality of life. Gut Liver 2017;11:370-376.

9. Ng WK, Wong SH, Ng SC. Changing epidemiological trends of inflammatory bowel disease in Asia. Intest Res 2016;14:111-119.

10. Ueno F, Nakayama Y, Hagiwara E, Kurimoto S, Hibi T. Impact of inflammatory bowel disease on Japanese patients' quality of life: results of a patient questionnaire survey. J Gastroenterol 2017;52:555-567. 